bone and had possibly opened the capsule of the joint at the same time. The X-ray picture elearly demonstrated the absence of fracture and the existence of a deep furrow with numerous fragments on each side. The X-ray apparatus also proved of the greatest practical utility in showing the displacement of fragments in gunshot fractures of the long bones, and enabled the surgeons to resort to timely measures to prevent vicious union. The fluoroscope has greatly added to the practical value of skiagraphy. In the light of our recent experience the X-ray has become an indispenseble diagnostic resource to the military surgeon in active service, and the suggestion that the chief surgeon of every army corps should be supplied with a portable apparatus and an expert to use it must be considered a timely and an urgent one.

\section{SPECIAL SENSES.}

Sources of Failure in Treating Lachrymal Obstructions.-(Journal of American Medical Association, October 10th, 1898). Dr. L. Connor of Detroit concludes that fewer failures will occur if the operator (1) recognizes and treats the constitutional disorder which caused the obstruction, e.g., syphilis, gout, rheumatism, or scrofula; (2) removes all eye-strain now generally accepted as a cause of -conjunctivitis and blepharitis, and consequently of lachrymal obstruction; (3) makes sure that the naris adjacent to the opening of the lachrymal duct are reudered healthy. The termination is as important as the beginning of a continuous tube when obstructed ; (4) perfects himself in faultless technique. Under this heading Dr. Connor insists on the division of the canaliculus being as limited as possible, and on its being so made as to leave the opening of the upper end of the lachrymal tube within the lachrymal lobe, inasmuch as a syphon will not act unless its upper end lies beneath the surface of the fluid it is expected to drain. The upper canaliculus should be chosen unless the stricture affects the lower. Gentle but firm use of probes is advocated only when antiseptic washings fail to remove the obstruction.

In the same journal Drs. Melville, Black and Reik advocate the use of large probes (up to Theobald's No. 16) in the treatment of stenosis of the lachrymal duct. In the subsequent discussion that ensued (in the section of ophthalmology at the 49th meeting of the American Medical Association, 1898) several well-known men condemned the use of such large probes $(4 \mathrm{~mm}$. diameter), and the majority agreed that it was better to use not as large probes as can be passed but as small ones as are adequate.

Quinine Amblyopia.-At the above-quoted meeting Dr. Ellett, of Memphis, Tenn, related a case in which, like many others noted recently in these columns, the reasons for regarding the amblyopia as due to quinine were anything but conclusive. The patient snoked cigarettes from the age of seven gradually increasing the number, until from 1890 to 1893 , he smoked from 40 to 50 a day. Then he knocked off a little. In 1894, when 30 years of age, he suffered from tertian malarial fever for a month and finally took 120 grains of quinine in 12 hours. The malarial organisms were routed, but five days later his sight became impaired; p. l. was lost the same day and not regained for three months. The two eyes were unequally affected, a relative ring scotoma being found in one and a central scotoma in the other. Ophthalmoscopically there was white optic atrophy with papery nerve head and attenuated vessels. The pupils were dilated but responded to light and to accommodation. There had been very slight improvement in vision up to 1898 .

At the same meeting a paper was read on Amblyopia from auto-intoxication by Dr. Young, Iowa, in which several cases were associated with manifest disorders of digestion, and regarded as due to auto-intoxication. Horner in 1873 believed that the chronic gastritis in alcoholics had as much to do with the amblyopia as the alcohol.
Sachs recently stated that it is not the nicotine or alcohol but compounds of these in the stomach which produce the retrobulbar neuritis of toxic amblyopia. In an interesting discussion which followed, men like Hotz of Chicago, Reynolds of Louisville, Conmor of Detroit and Wilder of Chicago, declared they had never seen a case of quinine amblyopia. Dr. Casey A. Wood, Chicago, said that he had obtained ample evidence of the auto-intoxication theory being true. In a recent series of experiments on dogs, amblyopia followed hypodermic injection of ptomaines. He also pointed out that tobacco does not set up changes in other nerves. This was controverted by Dr. Lautenbach, who pointed to the arrest of growth produced by tobacco and alcohol, to the now recognized tobacco-deafness, and to the effect upon the heart of the consumption of tobacco. He also asserted that Ceylon tea was displacing Chinese tea in America on account of its superior flavour and might be expected, as it is more powerful in its effects upon the nervous system (is it?) to largely increase the cases of amblyopia due to teadrinking.

Eucain B. as a local Anæsthetic in the Nose.-Dr. Somers (Ther.peutic Gazette, Sept. 15th, 1898) reports his results with this drug. Eucain hydrochloride (A) has been found unsatisfactory in its not shrinking engorged cavernous tissue in cases of hypertrophic rhinitis. A three-per cent. solution was used, the drug being dissolved in sterile water by heat, and applied to the parts desired by means of a cotton plug saturated with the drug. The mucosa of the inferior turbinated bone became as anæsthetic in eight minutes as it usually does with a four-per-cent. solution of cocain. The anæsthesia passed off quicker however in about 15 minutes. The Eucain B. solution deteriorated gradually, and though its value remained unimpaired for three weeks, by the end of the fourth it had lost its aniesthetic properties entirely. No constitutional $\in$ ffects were ever produced. As its action is slower and its anæs. thesia shorter than cocain, and as it has no shrinking action on the mucosa, Dr. Somers concludes that it is inferior in value to cocain, in spite of its being nontoxic, more rapid and less irritating than Eucain A.

\section{Aqueous Extract of Supra-renal Capsule} in Ophthalmic Practice.-(Kyle, of Indiana, in Ophthalmic Record, April 1898 quoted in the Therapeutic Gazette, September 1898.) Papers by various authors are epitomised. They confirm the wonderful therapentic effects of this extract, already drawn attention to in the columns of the Indian Medical Gazette, Vol. XXXIII, pp. 72 and 233. The extract is prepared from the medulla. Twelve grains represent one fresh suprarenal gland. A two to four per cent. solution is powerfully astringent, causes no smarting, and has no mydriatic or myotic effect No congestion follows the astringent effects. It has a decided hœmostatic action and prolongs and increases the effect of cocain. This is especially marked in operation on meibomian cysts where cocain alone is unsatisfactory. The extract is indicated in chronic trachoma, pannus, lachrymal inflammation, acute conjunctivitis, panophthalmitis and iritis. The solution may be sterilised and a few drops of carbolic acid added to preserve it. Perchloride of mercury can not be used with it; a fresh solution is better. Two or three drops are sufficient. F. P. MAYNARD, s.B., D.P.H.

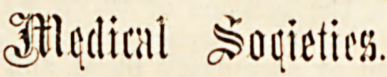

SOUTH INDIAN BRANCH OF B. M. A. Insomnia and the effects of Hypnotic Drugs. BY C. H. L. PALK, CAPTAIN, I.M.S.

IT having fallen to my lot to have been assoeiated for some time past with mental diseases in their various forms, $I$ have taken a special interest in the most common, and often the 